\title{
APLICAÇÃO DA TÉCNICA DE RECONCILIAÇÃO DE DADOS NO DIAGGNÓSTICO DAS MEDIÇÕES DE UMA MALHA DE DISTRIBUIÇÃO DE GÁS BFG*
}

\author{
Cláudio Antunes de Oliveira ${ }^{1}$ \\ Fernando da Silva Dummer² \\ Vinicius Tiago Lecco Rampinelli ${ }^{3}$ \\ Northon Torezani Cavazzonit
}

\section{Resumo}

Este trabalho tem como objetivo demonstrar uma aplicação prática da técnica de reconciliação de dados no diagnóstico das medições que compõe uma malha de distribuição de gás BFG - Blast Furnace Gas. Falhas comuns de medição tais como saturação e congelamento da indicação afetam a qualidade da informação e este tipo de detecção faz parte da proposta de diagnóstico. Será abordado o desenvolvimento de um algoritmo que implementa a técnica de reconciliação, bem como a elaboração de uma matriz de diagnóstico no InfoPlus.21. Os principais indicadores deste diagnóstico são armazenados na base de dados do InfoPlus.21 visando consultas históricas e monitoramento contínuo das medições. Os dados obtidos demonstraram que a técnica de reconciliação de dados pode contribuir para identificação de falhas e agregar confiabilidade às medições.

Palavras-chave: Confiabilidade; Diagnóstico; Reconciliação de dados.

\section{APPLICATION OF DATA RECONCILIATION TECHNIQUE FOR MEASUREMENTS DIAGNOSIS}

\section{Abstract}

This work proposes a practical application of data reconciliation technique in the diagnosis of the measurements that comprise a network of gas distribution BFG Blast Furnace Gas. Measurements with faults such as saturation or frozen indication can affect the quality of information and this type of detection is part of the proposed diagnosis. The development of an algorithm that implements this technique and also a diagnosis tool implemented on InfoPlus. 21 will be showed. The main indicators of this diagnosis are stored on InfoPlus.21 database to allow historical queries and continuous monitoring measurements. The results show that using this technique is possible to contribute with fault identification and aggregate reliability of the measurements.

Keywords: Reliability; Diagnosis; Data reconciliation.

1 Engenheiro Eletricista, Especialização, Especialista em Automação, Gerência de Informática e Automação de Processo, ArcelorMittal Tubarão, Serra, ES e Brasil.

2 Engenheiro Eletricista, Especialista em Automação, Gerência de Informática e Automação de Processo, ArcelorMittal Tubarão, Serra, ES e Brasil.

3 Engenheiro Eletricista, Mestre, Especialista em Automação, Gerência de Informática e Automação de Processo, ArcelorMittal Tubarão, Serra, ES e Brasil.

4 Graduando em Engenharia Elétrica, Estagiário, Gerência de Informática e Automação de Processo, ArcelorMittal Tubarão, Serra, ES e Brasil.

\footnotetext{
* Contribuição técnica ao $18^{\circ}$ Seminário de Automação e TI Industrial, 23 a 26 de setembro de 2014, São Paulo, SP, Brasil.
} 


\section{INTRODUÇÃO}

Com o advento de sistemas automatizados de coleta de dados representados por PLC's, sistemas supervisórios e PIMS - Plant Information Managment System o aspecto de se obter e encaminhar dados transformando-os em informações foi resolvido. Entretanto, quando tentamos utilizar os dados verificamos que os dados não obedecem a equações de balanço.

Por que um balanço de massas não fecha?

Porque os dados colhidos são muitas vezes inconsistentes.

As totalizações das entradas e saídas dos equipamentos de processo, considerando-se as acumulações, como tanques, pilhas de homogeneização de minérios etc., estão sempre numa situação de balanço matemático, mas as medidas coletadas não. Os erros são devidos a:

$\checkmark$ Erro de medição aleatório;

$\checkmark$ Instrumento descalibrado;

$\checkmark$ Modelamento inadequado;

$\checkmark$ Amostragem na freqüência incorreta;

$\checkmark$ Não linearidade do instrumento;

$\checkmark$ Densidade incorreta ou variando com temperatura;

$\checkmark$ Polarização de leitura do instrumento;

$\checkmark$ Leitura fora de faixa do instrumento;

$\checkmark$ Erro de transmissão do sinal, etc.

A maneira usual de se contornar este problema consiste em se atribuir maior grau de confiança para determinadas medidas baseado em algum conhecimento empírico do funcionamento da planta e de se alterar os dados artificialmente [1].

Diante do exposto, o objetivo principal deste trabalho é demonstrar de forma prática, um método matemático para se recalcular o valor mais provável para as medições de uma malha de distribuição de gás BFG, empregando-se uma técnica denominada reconciliação de dados.

A reconciliação de dados é um método de ajuste das variáveis medidas em um determinado processo, de modo a satisfazer certas restrições - tipicamente balanços de massa ou energia, respeitando-se a natureza estatística e metrológica de cada medição. A natureza estatística das medições resulta do fato de que toda medição obtida em um processo real possui uma incerteza inerente, o que implica em erros e imprecisões. Sabendo-se que as plantas industriais modernas possuem um elevado grau de automação e que diversas variáveis são medidas a taxas de amostragem freqüentes, pode-se lançar mão desta abundância (e provável redundância) de informação para interpretar, identificar e reduzir os erros de medição. Portanto, a técnica de reconciliação de dados pode ser definida como uma forma de aproximar as medições de seus valores verdadeiros, o que equivale a reduzir os erros de medição, valendo-se de redundâncias presentes no sistema [2]. A figura 1 ilustra um exemplo de reconciliação de dados. As medidas M1, M2 e M3 são denominadas medidas reconciliadas. Neste exemplo, a medida M1 foi a que recebeu o maior ajuste.

* Contribuição técnica ao 18`Seminário de Automação e TI Industrial, 23 a 26 de setembro de 2014, São Paulo, SP, Brasil. 

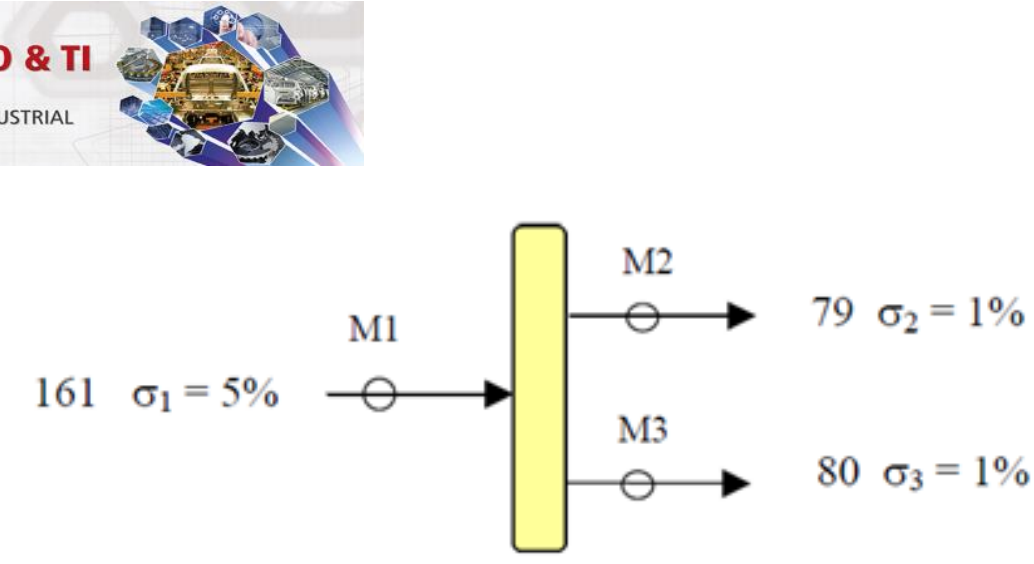

Medidas Reconciliadas

\begin{tabular}{|c|c|c|}
\hline \multicolumn{3}{|c|}{ Medidas Reconciliadas } \\
\hline M1 & M2 & M3 \\
\hline 159,0383 & 79,0189 & 80,0194 \\
\hline
\end{tabular}

Figura 1 - Exemplo de reconciliação de dados

A forma geral de um problema de reconciliação de dados pode ser representada conforme indicada na equação 1 ,

sujeito a

$$
\min _{x, u, p} \mathbb{F}\left(x^{M}, x\right)
$$

$$
\begin{gathered}
h(x, u, p)=0 \\
g(x, u, p) \leq 0 \\
x^{L} \leq x \leq x^{U} \\
u^{L} \leq u \leq u^{U} \\
p^{L} \leq p \leq p^{U}
\end{gathered}
$$

onde $\mathbb{F}$ é uma função objetivo a ser minimizada, $\boldsymbol{x}^{M}$ é o vetor das variáveis medidas no processo e $\boldsymbol{x}$ é o vetor dos valores reconciliados destas variáveis medidas. As variáveis não medidas (vetor $\boldsymbol{u}$ ) e os parâmetros (vetor $\boldsymbol{p}$ ) podem ser estimados. As restrições à minimização podem ser colocadas na forma de equações $(\boldsymbol{h})$ ou inequações $(\boldsymbol{g})$.

Tradicionalmente, a função objetivo $\mathbb{F}$ indicada na equação 2 , é a função soma dos quadrados ponderados,

$$
\mathbb{F}=\sum_{i=1}^{n} \frac{\left(x i^{M}-x i\right)^{2}}{\sigma i^{2}}
$$

onde $\boldsymbol{n}$ representa o número de variáveis medidas e $\boldsymbol{\sigma} \boldsymbol{i}^{2}$ representa a variância da iésima medição [2].

Assim, serão abordados também neste trabalho, o desenvolvimento de um algoritmo que implementa a técnica de reconciliação de dados por meio do método dos multiplicadores de Lagrange [2] e a elaboração de uma matriz de diagnóstico no InfoPlus.21 que visa auxiliar o monitoramento contínuo das medições pertencentes a malha de distribuição.

O texto está organizado em 4 seções. A seção 2 descreve os materiais e métodos enquanto que os resultados são abordados na seção 3. Para finalizar, a seção 4 apresenta as conclusões e trabalhos futuros.

* Contribuição técnica ao 18Seminário de Automação e TI Industrial, 23 a 26 de setembro de 2014, São Paulo, SP, Brasil. 


\section{MATERIAIS E MÉTODOS}

Este trabalho foi desenvolvido de acordo com as 5 etapas mostradas na figura 2, as quais serão detalhadas nos sub-tópicos seguintes. O software Microsoft Excel 2007 foi utilizado para coleta e tratamento dos dados e o software Matlab R2013a foi empregado para realização das simulações.

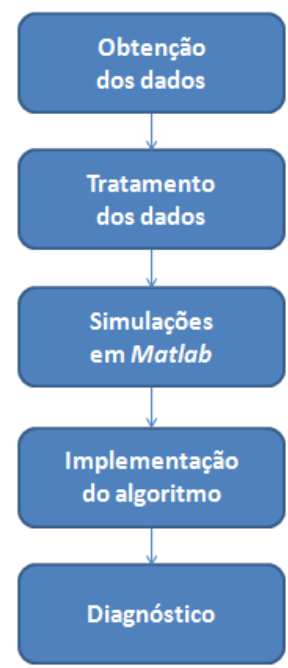

Figura 2 - Etapas do trabalho

\subsection{Obtenção dos Dados}

A massa de dados composta por 22 variáveis da malha de distribuição foi obtida diretamente do Sistema de Otimização de Processo de Nível 2. A massa de dados foi exportada desta plataforma para arquivos do tipo texto. Estas variáveis foram amostradas a uma freqüência de 60 s por um período de 6 meses, totalizando uma massa de dados composta por 259.200 minutos para realização das simulações em ambiente Matlab.

\subsection{Tratamento dos Dados}

Nesta etapa foi dedicada uma atenção especial a questão da falta de medição em determinados pontos da malha de distribuição. De acordo com a técnica de reconciliação de dados, a variável não medida é denominada variável observável [2]. Para estes casos, foi necessário estimar estas medições com o auxílio de outras medições presentes na malha de distribuição e restrições do processo.

\subsection{Simulações em Matlab}

Nesta etapa, foi desenvolvido no ambiente Matlab, um algoritmo para implementar o método dos multiplicadores de Lagrange, que foi o método de minimização escolhido [1]. Um fragmento deste algoritmo pode ser visualizado na figura 3.

* Contribuição técnica ao 18Seminário de Automação e TI Industrial, 23 a 26 de setembro de 2014, São Paulo, SP, Brasil. 


\section{AUTOMAÇÃO \& TI}
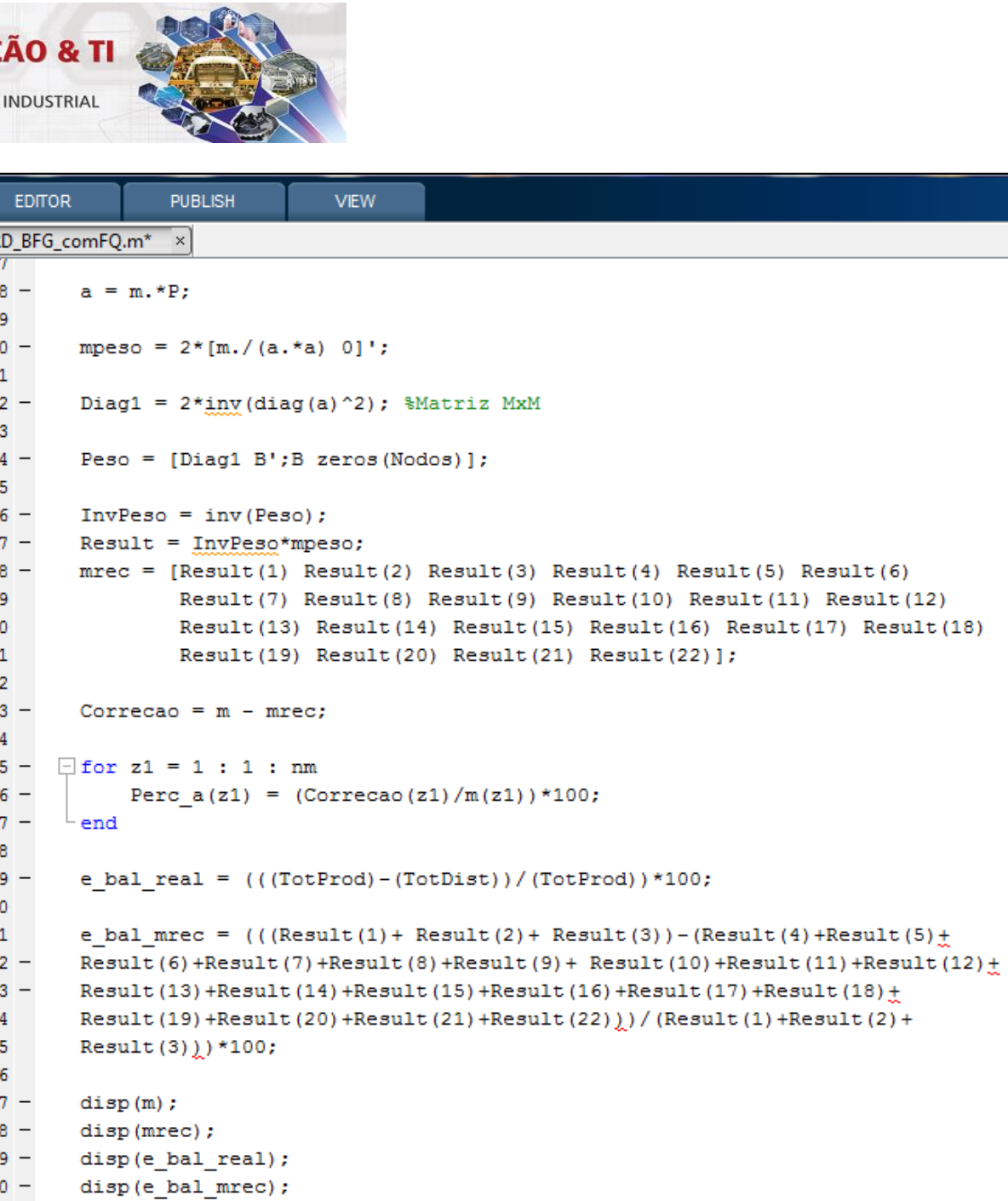

Figura 3 - Fragmento do código em Matlab

A simulação off-line realizada com a massa de dados coletada possibilitou sedimentar o conhecimento a respeito da técnica de reconciliação de dados bem como validar a metodologia empregada.

\subsection{Implementação do Algoritmo}

Mediante a conclusão das simulações, foram desenvolvidos no Sistema de Otimização de Processo de Nível 2, dois algoritmos em linguagem Fortran para implementar a técnica de reconciliação de dados. A figura 4 mostra as fases de execução destes algoritmos.

\section{Carrega as medições da malha de distribuição.}

\section{Valida as medições}

3.Reconstrói as medições
necessárias.

\section{Executa a reconciliação.}

\section{Gera o diagnóstico.}

6. Envia os resultados ao InfoPlus. 21 via comunicação FTP.

Figura 4 - Etapas de execução do algoritmo

* Contribuição técnica ao $18^{\circ}$ Seminário de Automação e TI Industrial, 23 a 26 de setembro de 2014, São Paulo, SP, Brasil. 
As figuras 5 e 6 mostram um fragmento dos algoritmos util_recon_dados_bfg.for e recon_dados.for, respectivamente. O primeiro executa as etapas de 1 a 3 , enquanto o segundo executa as etapas de 4 a 6.

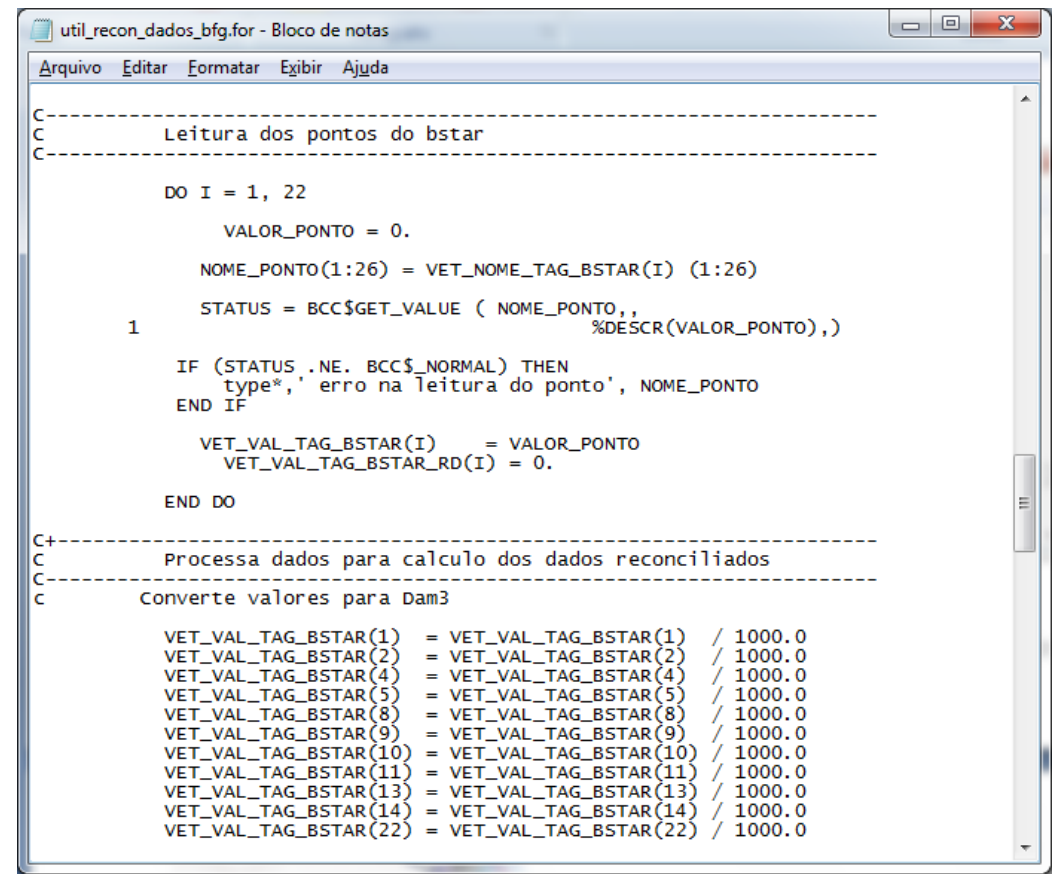

Figura 5 - Algoritmo auxiliar

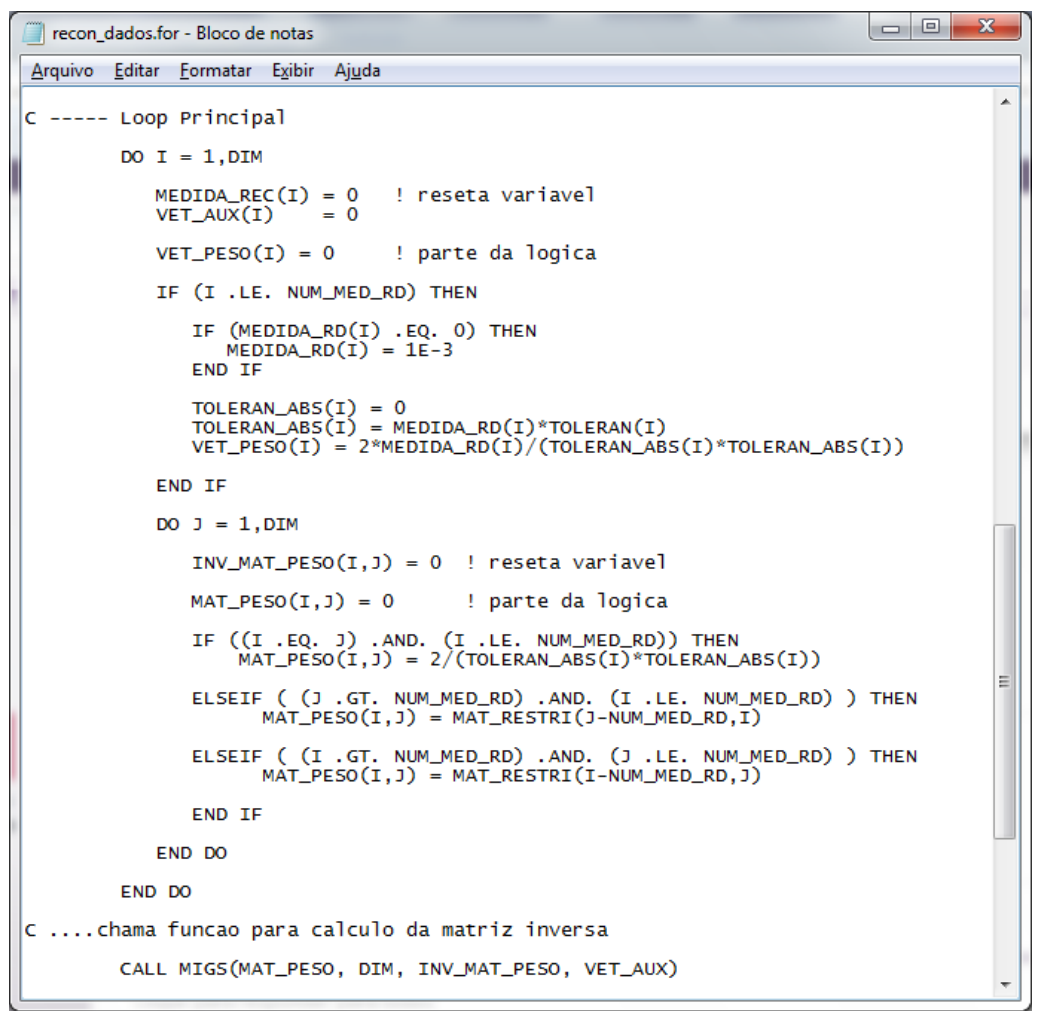

Figura 6 - Algoritmo principal

$\mathrm{Na}$ fase 5 são geradas as informações utilizadas no diagnóstico. O subitem 2.5 abordará esta questão. Já a fase 6 , encarrega-se de enviar todos os resultados gerados para o Infoplus.21 via comunicação FTP, visando armazená-los em uma base de dados. Com isso, é possível consultar os dados históricos de ajuste de cada

* Contribuição técnica ao 18Seminário de Automação e TI Industrial, 23 a 26 de setembro de 2014, São Paulo, SP, Brasil. 


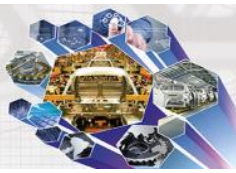

medição ou avaliar, por exemplo, o erro do balanço em tempo real. A figura 7 ilustra alguns dados históricos armazenados no InfoPlus.21.

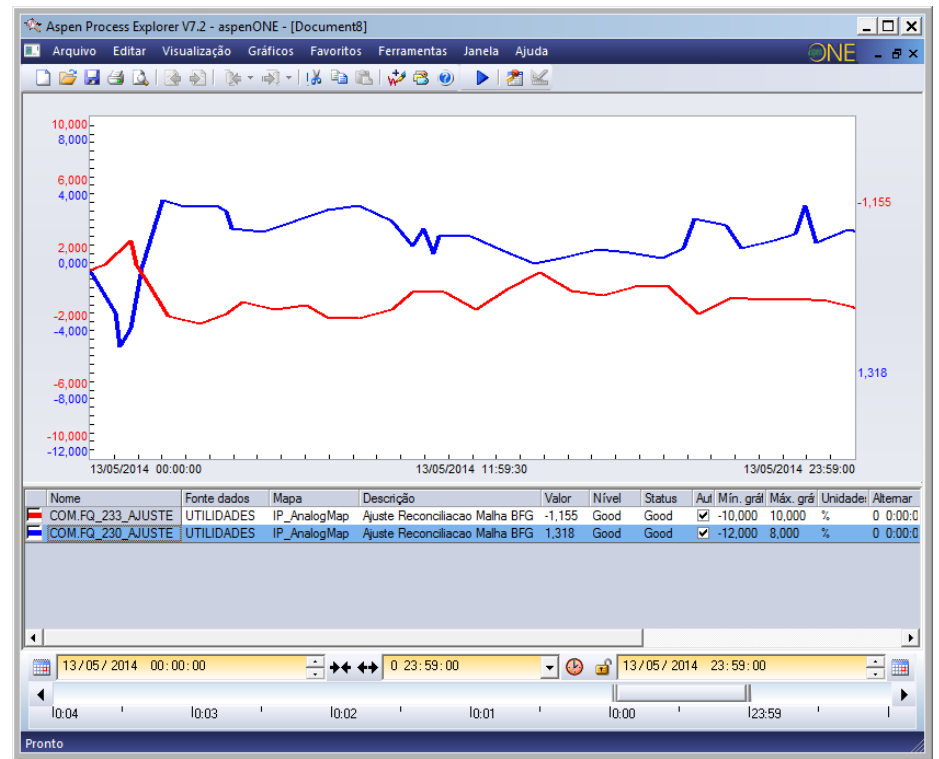

Figura 7 - Histórico do percentual de ajuste

\subsection{Diagnóstico}

A partir das informações geradas pela técnica de reconciliação de dados como o percentual de ajuste das medições e a própria medida reconciliada, foi gerada uma matriz de diagnóstico, visando demonstrar o diagnóstico das medições pertencentes a malha de distribuição em uma interface de fácil interpretação. O foco deste diagnóstico é a equipe de manutenção, pois através dele é possível identificar facilmente, qual medição está impactando no balanço da malha de distribuição do gás BFG. A figura 8 ilustra a matriz de diagnóstico elaborada.

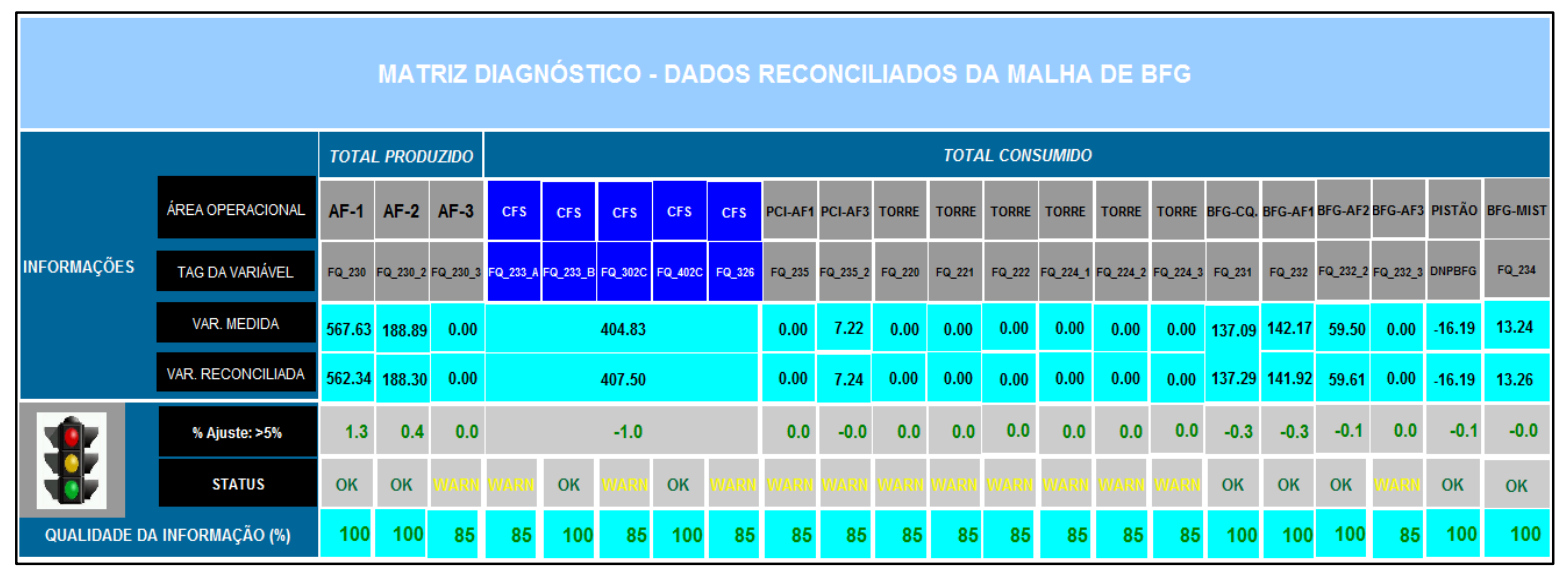

Figura 8 - Matriz de diagnóstico

\section{RESULTADOS E DISCUSSÃO}

O principal resultado obtido através da implantação da técnica de reconciliação de dados foi sem dúvida a geração do diagnóstico individual para cada medição pertencente à malha de distribuição de gás. Os diagnósticos obtidos são:

* Contribuição técnica ao $18^{\circ}$ Seminário de Automação e TI Industrial, 23 a 26 de setembro de 2014, São Paulo, SP, Brasil. 
- \% Ajuste (percentual de ajuste): é o principal indicador da matriz, pois através dele, identifica-se qual medição sofreu maior ajuste. Este indicador pode apontar, por exemplo, uma falha desta medição que deve ser analisada pela equipe de manutenção;

- STATUS (status da medição): este diagnóstico aponta três condições para as variáveis:

1. OK: a variável está normal;

2. NOK: a variável apresenta períodos prolongados de congelamento, zeramento ou saturação;

3. $B A D$ : a variável apresenta a combinação das condições NOK, por exemplo, a variável encontra-se congelada na faixa superior do range.

- Qualidade da informação: este diagnóstico aponta três condições para a qualidade da informação de cada variável:

1. Verde (85 a 100\%) - se a variável apresentar status OK e percentual de ajuste $<5 \%$;

2. Amarelo (75 a 84\%) - se a variável apresentar status NOK e percentual de ajuste $<5 \%$;

3. Vermelho $(<75 \%)$ - se a variável apresentar status NOK ou BAD e percentual de ajuste $>5 \%$.

A informação da variável reconciliada, nomeada pela matriz de diagnóstico como VAR. RECONCILIADA, refere-se ao novo valor estimado para a variável medida após execução do algoritmo de reconciliação, levando-se em consideração as restrições da malha de distribuição.

O diagnóstico status da medição demonstrou-se eficiente na identificação de tendências de falhas tais como congelamento, saturação e zeramento da indicação.

O diagnóstico percentual de ajuste por sua vez, possibilitará uma análise mais abrangente do erro do balanço, como, por exemplo, correlacionando a tendência deste indicador para um determinado grupo de medições com condições de processo, permitindo com isso identificar melhorias que auxiliem na otimização do processo de distribuição de gás BFG para as unidades consumidoras.

\section{CONCLUSÃo}

Este trabalho apresentou a aplicação da técnica de reconciliação de dados em tempo real, para uma malha de distribuição de gás BFG, através do desenvolvimento de um algoritmo em linguagem Fortran.

A integração entre o Sistema de Otimização de Processo de Nível 2 - plataforma onde o algoritmo é executado e o InfoPlus.21, permitiu o desenvolvimento da matriz de diagnóstico em um ambiente totalmente voltado para as equipes de manutenção com todas as facilidades de análise disponíveis. Para o futuro, estuda-se a implantação deste algoritmo no próprio Infoplus.21 utilizando o recurso da ferramenta SQLPIUS.

Como a redundância de medição é um fator importante para a técnica de reconciliação de dados, principalmente para a identificação de erros grosseiros a qual não foi abordada neste trabalho, estuda-se no futuro, resolver os problemas de redundância identificados nesta malha de distribuição tomada como estudo de caso, visando eliminar a etapa de reconstrução de medições.

\footnotetext{
* Contribuição técnica ao 18Seminário de Automação e TI Industrial, 23 a 26 de setembro de 2014, São Paulo, SP, Brasil.
} 


\section{Agradecimentos}

Agradeço aos colegas da ArcelorMittal Tubarão dos setores de Automação e Energia que apoiaram o desenvolvimento deste trabalho.

\section{REFERÊNCIAS}

1 Reconciliação e análise de qualidade de dados. [acesso em 15 jan. 2014] 1-16. Disponível em:

http://www.cpdee.ufmg.br/ seixas/Especializacao/Download/DownloadFiles/Reconcilia cao.PDF

2 Feldman RN. Reconciliação de dados em tempo real para monitoração e detecção de falhas em terminal de transporte e armazenamento de derivados de petróleo [dissertação mestrado]. Universidade Federal do Rio de Janeiro; 2007. 